\title{
Large eddy simulations of targeted electromagnetic control of buoyancy-driven turbulent flow in a slender enclosure
}

Received: 19 October 2008 / Accepted: 2 June 2009 / Published online: 10 July 2009 (C) The Author(s) 2009. This article is published with open access at Springerlink.com

\begin{abstract}
We conducted a large eddy simulation (LES) of a locally applied electromagnetic control of turbulent thermal convection of an electrically conductive fluid (electrolyte solution) inside of a slender enclosure. Generic configurations, consisting of two or three magnets of opposite polarities located below the lower wall, and two oppositely charged electrodes along the side walls, are considered. The neutral situation (pure thermal convection) is selected to be in turbulent regime at $R a=10^{7}, \mathrm{Pr}=7$. A magnetically extended Smagorinsky type model for the subgrid turbulent stresses and a simple-gradient diffusion model for the subgrid turbulent heat fluxes are used. Different intensities of applied DC current through electrodes are imposed. The effects of the resulting Lorentz force on flow, turbulence reorganisation and wall-heat transfer are analysed. It is demonstrated that significant flow and turbulence structure reorganisation takes place in the proximity of the lower horizontal wall and in the central parts of the enclosure-even for weak DC current of $I=1 \mathrm{~A}$. Significant turbulence increase, generated by the elevated electromagnetic mixing, produced significant enhancements of the wall-heat transfer-up to $70 \%$ for the 2-magnet configuration.
\end{abstract}

Keywords Heat transfer control · Turbulence $\cdot$ Electromagnetic fields $\cdot$ Lorentz force $\cdot$ LES

PACS 47.27.i, 47.65.-d, 47.85.-g

\section{Introduction}

For electrically conductive fluids, fundamental insights into the interactions between fluid flow, heat transfer and imposed electromagnetic fields are of great importance for better understanding and possible control and optimization of many industrial and technological applications. These include various metallurgical processes, the dynamics of crystal growth, or new designs for future generations of fusion reactors, etc., Moreau [33], Davidson [8], Barleaon et al. [2]. This complex multi-physics (fluid dynamics/electromagnetism) phenomenon is even more difficult when turbulent flow regimes are considered. In such cases, the direct and indirect interaction between fluctuating hydrodynamical (velocity, pressure, temperature) and electromagnetic (magnetic field, electric potential, electric current) variables should be taken into account. Despite the impressive development of experimental techniques in past decades, simultaneous measurements of both hydrodynamical and electromagnetic variables and their (cross) correlations are still a challenging task. At present, the only way to extract full three-dimensional flow and electromagnetic fields and to dynamically track spatial evolutions of these fields and their interactions, is to perform numerical simulations.

Communicated by O. Zikanov 
In the present study, we report on the application of Large Eddy Simulations approach in calculating a generic case of locally applied electromagnetic forcing in order to control flow, turbulence and heat transfer.

First, a short overview of some of the previous studies in literature dealing with electromagnetic flow control is given. This Section is divided in three major application groups: electromagnetic control of pressure-driven flows, purely electromagnetically driven flows and finally, electromagnetic control of thermal buoyancy driven flows.

\subsection{Electromagnetic control of pressure-driven flows}

Starting from the pioneering work of Gailitis and Lielausis [10], who proposed a simple method for seawater drag reduction, using electrodes and magnetic elements, numerous experimental studies involving similar principles have been reported in literature.

An extended experimental investigation of a salt water turbulent boundary layer in a simple channel subjected to a stream-wise Lorentz force was reported in Henoch and Stace [15]. Single-component velocity field measurements, photographic flow visualisations as well as mean and fluctuating wall shear stress measurements were performed. Different flow regimes, characterised by different intensities of imposed forcing including strong, moderate and weak interactions, were presented. The main conclusions were that for strong interactions, an increase in the mean wall shear and in the turbulence intensity were observed. In contrast to that, for moderate interactions only fluctuating quantities were affected and $30 \%$ suppression (compared to their neutral case values) of the wall shear stress and intensity of turbulence was observed.

Weir et al. [46] reported experimental results on the prevention of flow separation of an electrolyte with a relatively low electric conductivity, by applying a stream-wise, wall-parallel Lorentz force acting on the suction (upper) side of inclined flat plates and hydro-foils. The fully developed turbulent flow regime $\left(1.2 \times 10^{5} \leq\right.$ $\left.R e \leq 3.7 \times 10^{5}\right)$ was considered for different intensities of applied electric currents, $(50 \leq I \leq 1,000 \mathrm{~A})$. Signals of drag, lift and pitching moment were recorded together with intensities of the applied Lorentz forces along the hydrofoil surface. For sufficiently strong forcing, a significant lift increase (up to 60\%) was observed for all considered angles of approaching flow.

Experimental studies by Dattarajan and Johari [7] addressed the control of a separating turbulent boundary layer $\left(1.1 \times 10^{4} \leq R e \leq 2.3 \times 10^{4}\right)$ of sea water along a two-dimensional hydrofoil with electromagnetic actuators. The effects of time-periodic span-wise oriented Lorentz force on flow separation, reattachment and drag reduction were investigated. The velocity fields were measured by using particle image velocimetry (PIV) in order to evaluate the performance of the imposed forcing. It was observed that above a certain amplitude of imposed forcing, in contrast to the neutral situation, complete reattachment of the flow over the flap occurred. It was concluded that the span-wise Lorentz forces generated stream-wise vorticity that was responsible for flow reattachment.

In contrast to all above-mentioned isothermal studies, Inaoka et al. [17] additionally investigated possibilities to affect the heat transfer in a backward-facing step flow in a duct by applying miniature electromagnetic actuators. For laminar flows, spatial variations of the local heat transfer along the bottom walls were measured by thermo-sensitive liquid crystal sheet. It was concluded that electromagnetic actuators significantly reduced the heat transfer deterioration in comparison with a neutral situation.

\subsection{Electromagnetically-driven flows}

Honji et al. [16] presented a visualisation study of a two-dimensional laminar electromagnetically driven multivortex flow in a shallow tank. Electromagnetic driving was provided by combining five permanent magnets with alternating magnetic polarities placed under the tank and arranged symmetrically with respect to its centre, and electrodes that were submerged in the fluid at the left and right ends of the tank. Flow patterns in the form of cellular vortices were visualised. A relation for the middle-vortex length as a function of the intensity of electromagnetic forcing was proposed.

Rossi et al. [40,41] addressed electromagnetically driven steady quasi-two-dimensional laminar flows with multi-scale point topology. The authors introduced an interesting concept of imposed electromagnetic forcing originating from the fractal-structure based geometry of the permanent magnets with opposite polarities. Flow visualisations using both dye and PIV were presented. The structure of the stagnation-points was investigated together with energy spectra. 
Experimental investigations of freely decaying quasi two-dimensional turbulence in a thin layer of electrolyte subjected to electromagnetic forcing were presented in Cardoso et al. [4]. Particle image velocimetry techniques were used to determine instantaneous velocity fields and corresponding vorticity and stream function.

Verdoold et al. [44,45] performed LIF flow visualisations of an electromagnetically driven flow inside a rectangular tank. Different generic setups, including 2- and 3-magnet configurations with different orientations with respect to the imposed electric DC currents, were analysed. In contrast to the previously mentioned studies, of Honji et al. [16] and Rossi et al. [40,41], Verdoold et al. [45] applied electric currents that were sufficiently strong (up to $I=10 \mathrm{~A}$ ) to locally create transient and turbulent flow regimes. Experimental studies were based on dye visualisations similar to that presented in Honji et al. [16], but without velocity measurements.

Kenjereš et al. $[29,30]$ continued this investigation by presenting combined experimental and numerical studies of the setup of Verdoold et al. [44]. In this study, velocity measurements by PIV were also presented. In addition, large eddy simulation (LES) were performed and compared to the experimental results. Good agreement between velocity profiles at different locations was obtained for both magnetic configurations for all considered DC currents.

\subsection{Electromagnetic control of thermal buoyancy-driven flows}

Cioni et al. [6] investigated the effects of a vertical uniform magnetic field on Rayleigh-Bénard convection in a cylindrical geometry. A liquid metal (mercury) was used as the working fluid because of its high electric conductivity. Because of that, the imposed magnetic field could create sufficiently strong induced electrical currents and resulting Lorentz force able to affect the fluid motion, i.e. there is no need to impose external electric currents. By heating the lower wall, strong buoyancy-driven motions were generated with Rayleigh numbers up to $R a=10^{9}$. On such well-developed turbulent convection, a uniform vertically oriented magnetic field of different intensities (tunable from 0 to $4 \mathrm{~T}$, corresponding to Hartmann numbers up to 1,000) was applied. It was observed that application of a magnetic field increased the critical convective threshold, indicating suppression of fluid motion. A strong decrease in Nusselt number was observed for sufficiently strong magnetic fields.

Similarly, Burr and Müller [3] experimentally investigated the influence of a vertical uniform magnetic field on turbulent convection of a low Prandtl liquid metal $(\mathrm{Pr}=0.02)$ (sodium potassium alloy) in a rectangular box. An intermediate range of Rayleigh $\left(10^{3} \leq R a \leq 10^{5}\right)$ and Hartmann $(0 \leq H a \leq 100)$ numbers was considered. Application of a magnetic field reduced the integral heat transfer. Also, local time-dependent temperatures were recorded by using four-element temperature probes placed in the middle of the domain. Fluctuations of the temperature field were significantly damped by the imposed magnetic field.

Hanjalić and Kenjereš [12] presented numerical simulations of reorganisation of the turbulence structure in turbulent convection subjected to an external uniform vertical magnetic field. Single-point subscale closures for turbulent stress and heat flux were employed in a transient RANS approach. The practical significance of such an approach lies in its capability to predict accurately the local wall-heat transfer by using a moderate numerical mesh. In this approach, the unresolved motion (in that study modelled by three-equations $k-\varepsilon-\theta^{2}$ closure) covers a significantly larger part of the turbulence spectrum (compared to LES), whereas the large eddy deterministic structure (mean motion) was fully resolved by the numerical mesh. The results of the numerical simulation for strong magnetic field $(H a=100)$ showed the appearance of a stable stratified interior, in accordance with experimental observations of Cioni et al. [6]. Also, thermal plumes were much more elongated in the vertical direction and horizontal movement of the boundary layers was significantly suppressed for situations with an imposed magnetic field.

Kenjereš and Hanjalić [23] performed numerical simulations of laminar and turbulent thermal convection subjected to an external uniform magnetic field of different orientations and strengths. The T-RANS approach was applied for simulations in turbulent flow regimes. The algebraic turbulent heat-flux model for unresolved scales was extended to include Prandtl-number-dependent molecular dissipation of heat flux. Simulations of turbulent Rayleigh-Bénard convection covered an extended range of Rayleigh $\left(10^{5} \leq R a \leq 10^{9}\right)$ and Hartmann $(0 \leq H a \leq 500)$ numbers, including longitudinal and perpendicular magnetic field orientations. For transversal magnetic field orientation (aligned with vertical direction) simulations showed good agreement with integral heat transfer suppression observed experimentally by Burr and Müller [3] and Cioni et al. [6]. For longitudinal magnetic field orientation (aligned with span-wise direction), a distinct two-dimensional flow reorganisation was observed. After reaching a two-dimensional state, the heat-transfer suppression was stopped. A further increase of imposed magnetic field strength was without any impact on heat transfer, i.e. a saturation point was reached. 


\subsection{Present contribution}

The present contribution represents a new combination of turbulent thermal convection subjected to external non-uniform electromagnetic fields. The non-uniformity of the imposed Lorentz force is imposed by combining permanent magnets of opposite polarities with oppositely charged electrodes. In that respect, the present study represents a continuation of our previous studies presented in Kenjereš et al. [29,30]. The first novel element is in the activation of the turbulent heat transfer, i.e. a background state of thermal convection in fully developed turbulent regime at $\operatorname{Ra}=10^{7}, \operatorname{Pr}=7$ is selected (where $\operatorname{Ra}=\beta g \Delta \operatorname{TPr} H^{3} / \nu^{2}, \operatorname{Pr}=v / a$ ). The second element is in using a magnetically extended subgrid closure for turbulent stress in the framework of an LES approach (in contrast to the previous T-RANS based approach). The third element is in the locally distributed Lorentz force-primarily focused in boundary layers along the thermally active horizontal walls.

The flow is confined in a $L: D: H=4: 4: 1$ enclosure, with dimensions of $L=0.6 \mathrm{~m}, D=0.6 \mathrm{~m}$, $H=0.155 \mathrm{~m}$, that represents exactly the experimental setup investigated in Verdoold et al. $[44,45]$ and Kenjereš et al. [29,30]. The electric conductivity of the working fluid is $\sigma=5.5 \mathrm{~S} / \mathrm{m}$, corresponding to water with $7 \%$ electrolyte $\left(\mathrm{Na}_{2} \mathrm{SO}_{4}\right)$ solution. Here, we will just mention the basic geometrical parameters. The permanent magnets are in the form of rectangular blocks with dimensions $0.084 \times 0.040 \times 0.040 \mathrm{~m}^{3}$ that are arranged in line with alternating polarities, providing magnetic fields of $1 \mathrm{~T}$ in the proximity of the lower horizontal wall. The electric field is generated by supplying a tunable DC current $(0 \leq I \leq 10 \mathrm{~A})$ through two opposite-charged electrodes mounted in the upper corners of the side walls. The lower horizontal wall is heated and the upper wall is cooled — both with uniform constant temperatures. The side walls are kept insulated, and consequently, no-slip adiabatic boundary conditions are imposed for all side walls.

\section{Equations and subgrid closure}

The equations describing the motion of an incompressible electrically conductive fluid in the turbulent flow regime subjected to both thermal buoyancy and Lorentz force, consist of the conservation of momentum and heat

$$
\begin{gathered}
\frac{\partial\left\langle U_{i}\right\rangle}{\partial t}+\left\langle U_{j}\right\rangle \frac{\partial\left\langle U_{i}\right\rangle}{\partial x_{j}}=\frac{\partial}{\partial x_{j}}\left(v \frac{\partial\left\langle U_{i}\right\rangle}{\partial x_{j}}-\tau_{i j}\right)-\frac{1}{\rho} \frac{\partial\langle P\rangle}{\partial x_{i}}-\underbrace{\beta g_{i}\left(\langle T\rangle-T_{R E F}\right)}_{F_{B}}+\underbrace{\frac{1}{\rho} \varepsilon_{i j k}\left\langle J_{j}\right\rangle\left\langle B_{k}\right\rangle}_{F_{L}} \\
\frac{\partial\langle T\rangle}{\partial t}+\left\langle U_{j}\right\rangle \frac{\partial\langle T\rangle}{\partial x_{j}}=\frac{\partial}{\partial x_{j}}\left(\frac{v}{\operatorname{Pr}} \frac{\partial\langle T\rangle}{\partial x_{j}}-\tau_{\theta j}\right)
\end{gathered}
$$

together with the divergency-free conditions for velocity $\left(\left\langle U_{i}\right\rangle\right)$, magnetic field $\left(\left\langle B_{i}\right\rangle\right)$ and for the total electric current $\left(\left\langle J_{i}\right\rangle\right)$ :

$$
\frac{\partial\left\langle U_{i}\right\rangle}{\partial x_{i}}=0, \quad \frac{\partial\left\langle B_{i}\right\rangle}{\partial x_{i}}=0, \quad \frac{\partial\left\langle J_{i}\right\rangle}{\partial x_{i}}=0 .
$$

Here, "〈 $\rangle$ " stands for the filtered (spatially averaged over a control volume) variables in a Large Eddy Simulation (LES) approach and $F_{B}$ and $F_{L}$ represent the thermal buoyancy (in the Boussinesq approximation with $T_{R E F}$ the reference temperature) and the Lorentz force, respectively.

Expression in the subgrid turbulence contributions in the momentum and temperature equations $\left(\tau_{i j}\right.$ and $\left.\tau_{\theta i}\right)$ must be provided in order to have a fully closed system of equations. The subgrid turbulent stress $\left(\tau_{i j}\right)$ and subgrid turbulent heat flux $\left(\tau_{\theta i}\right)$ are evaluated from the magnetically extended Smagorinsky [37] model of Shimomura [36] and a simple gradient diffusion of temperature, Kenjereš and Hanjalić [25], respectively:

$$
\begin{aligned}
\tau_{i j} & =\frac{1}{3} \tau_{k k} \delta_{i j}-v_{t}\left\langle S_{i j}\right\rangle, \quad\left\langle S_{i j}\right\rangle=\frac{1}{2}\left(\frac{\partial\left\langle U_{i}\right\rangle}{\partial x_{j}}+\frac{\partial\left\langle U_{j}\right\rangle}{\partial x_{i}}\right), \quad v_{t}=v_{s} \cdot \exp \left[\frac{-\frac{\sigma}{\rho}\left(C_{m} \Delta\right)^{2}\left|B_{0}\right|^{2}}{v_{s}}\right] \\
v_{s} & =\left(C_{s} \Delta\right)^{2}\left(\left\langle S_{i j}\right\rangle\left\langle S_{i j}\right\rangle\right)^{1 / 2}, \\
C_{s} & =C_{s 0}\left[1-\exp \left(\frac{-x_{i}^{N+}}{25}\right)\right]^{2}, \quad\left|B_{0}\right|=\sqrt{\left\langle B_{i}\right\rangle^{2}}, \quad \tau_{\theta i}=-\frac{v_{t}}{P r_{t}} \frac{\partial\langle T\rangle}{\partial x_{i}}
\end{aligned}
$$


Note that the van Driest [43] damping function is used since a wall-bounded enclosure is simulated. Here, $x_{i}^{N+}$ represents the non-dimensional wall distance from the nearest wall. The subgrid turbulence model constants are $C_{s 0}=0.1$ and $C_{m}=1.4$. The turbulent Prandtl number in the subgrid turbulent heat flux expression is assumed to be constant too; $P r_{t}=0.86$. The characteristic spatial filter $\Delta$ in the framework of a finite volume discretisation scheme used in this study is calculated as $\Delta=\Delta V^{1 / 3}$, where $\Delta V$ is the control volume.

\section{Numerical method}

The numerical discretisation of the set of Eqs. (1)-(4) is performed by using a finite-volume solver for general (non-orthogonal) structured multi-block geometries, Kenjereš and Hanjalić [20,23,24,27,28], Kenjereš et al. [26]. All variables are located in the centres of control volumes (CVs) and the Rhie-Chow interpolation [35] and SIMPLE algorithm are used for coupling between velocity and pressure fields. Both convective and diffusive terms in the momentum and temperature equations are represented by a second-order central differencing scheme (CDS). The time-dependent terms are discretised by a fully-implicit second-order three-consecutive time-steps scheme, Ferziger and Perić [9].

The simulation domain was represented by $182 \times 182 \times 92 \mathrm{CVs}$, clustered in the proximity of the thermally active side walls. The typical averaged values of the non-dimensional wall distance was $x_{i}^{N+} \approx 0.5$. The non-uniform mesh distribution is designed to fully resolve the estimated flow and turbulence scales in the proximity of the horizontal and side walls, i.e. $\Delta \approx(1-2) \eta_{K}$, where $\eta_{K}=\left(v^{3} / \varepsilon\right)^{1 / 4}$ is the Kolmogorov length scale. The dissipation rate of the turbulent kinetic energy is estimated from the following expression: $\varepsilon \approx\left|\left\langle U_{i}\right\rangle\right|_{\max }^{3} / H$. Special attention is given to the proper resolution of the steep temperature gradients in the proximity of the horizontal walls. In accordance with Grötzbach [11], 5-10 CVs are used for accurate representation of the thermal boundary layers along horizontal walls. The central part of the simulated domain is represented by a coarser mesh with $\Delta \approx(5-10) \eta_{K}$, which should be sufficient for a LES approach. This is confirmed by monitoring the maximum ratio between turbulent and molecular viscosity, which was $v_{t} / v<0.1$ even for the highest applied electric current of $I=10 \mathrm{~A}$ in the 2-magnet configuration. Typical values of the time-steps are selected to ensure that maximum CFL number will be 0.5 . The simulations are progressed in time until fully statistically converged fields are obtained. Between $5 \times 10^{4}$ and $7.5 \times 10^{4}$ instantaneous fields are used to obtain representative statistics of the second-moments. The numerical solver can be run in parallel mode using the MPI domain-decomposition directives. The present simulations were performed by using 16-32 CPUs on a IBM Power 6 system achieving ideal speed-up scaling.

\section{Results and discussion}

\subsection{Electromagnetic field distribution}

The magnetic and electric field distributions are calculated from a simplified set of Maxwell's equations by applying the Biot-Savart law for permanent magnets and electrodes by using the semi-analytical solutions as proposed in Akoun and Yonnet [1]. This was possible since the imposed electric and magnetic field strengths were constant in time. In addition, because the induced electric current is significantly smaller than its conductive counterpart, the total electric current can be simply written as $\left\langle J_{i}\right\rangle=\sigma\left\langle E_{i}\right\rangle$. This will give a Lorentz force that is constant in time, i.e. totally independent from the underlying fluid flow. This implies that the Lorentz force for the specific numerically discretised geometry can be calculated only once prior starting the fully coupled simulations.

Characteristic distributions of magnetic fields, for considered 2- and 3-magnet configurations, are shown in Figs. 1 and 2. Here, we show the superimposed magnetic flux lines with contours of particular magnetic field components in the central vertical planes $(x=0.3 \mathrm{~m}, y=0.3 \mathrm{~m})$ and in a horizontal plane in the proximity of the lower wall, $(z=0.001 \mathrm{~m})$. It can be seen that the highest concentration of magnetic flux lines is located at the edges of the magnets. For these magnetic configurations, the profiles of the resulting Lorentz force components in the central vertical planes, for different distances from the lower wall, and applied DC current of $I=1 \mathrm{~A}$, are shown in Fig. 3. It is noted that for the 2-magnet configuration, the vertical Lorentz force $\left(F_{z}\right)$ component is zero in the central vertical plane. This is the reason why the 2-magnet configuration that creates strong swirling (vortical) flow pattern in the central part of the tank, will be referred to as "shear-forcing". In contrast to that, the 3-magnet configuration generates significant Lorentz force components in both horizontal 

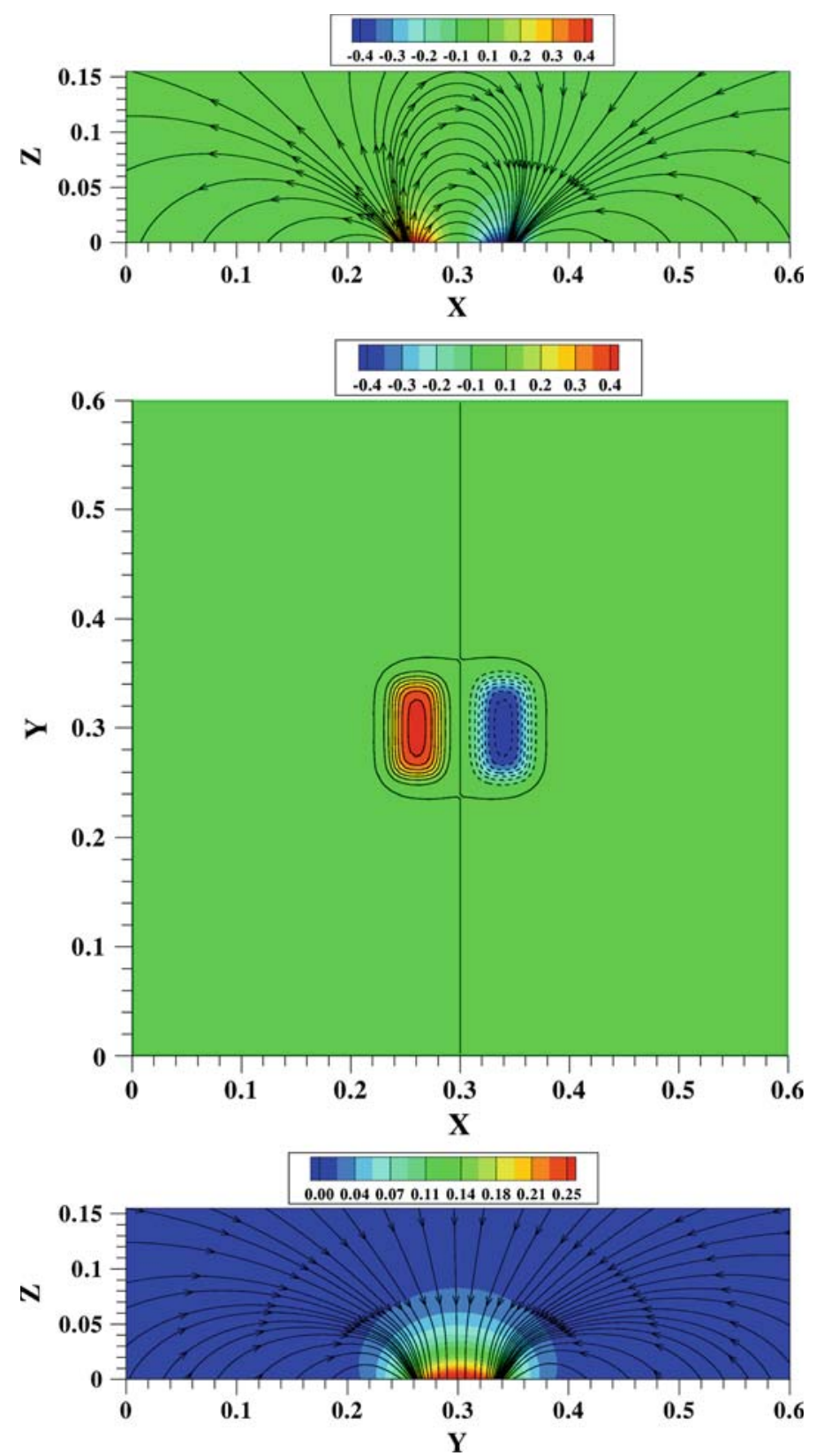

Fig. 1 The 2-magnets configuration: Distributions of the magnetic-flux lines and superimposed contours of the magnetic field components (in T): top $B_{z}$ in the $y / D=0.5$ plane, middle $B_{z}$ in the $z=0.001 \mathrm{~m}$ plane, bottom $B_{x}$ in the $x / L=0.5$ plane. The imposed electric field lines are distributed from left to right along the $x$-direction

and vertical directions. This configuration will be referred to as "forcing-in-and-out" configuration, since it primarily affects the boundary layers in the proximity of the lower wall. In order to illustrate these forcing effects, the velocity vectors and contours of the velocity magnitude in a horizontal plane in the proximity of the lower wall are shown in Fig. 4. These long-time averaged velocity fields are results for the isothermal case with activated electromagnetic forcing. The 2-magnet configuration imposes a relatively simple flow pattern consisting from two parallel opposite jets, Fig. 4(left). The 3-magnet configuration generates a complex flow pattern with counter-rotating vortices along the central line. The vertical velocity components in the central horizontal plane $(z / H=0.5)$-for both configurations-are shown in Fig. 4(below). The vertical velocity distribution in the 2-magnet configuration is characterised with distinct central and wall regions. In the central part of the tank, a single area with one large negative vertical velocity region and four smaller diagonally located 

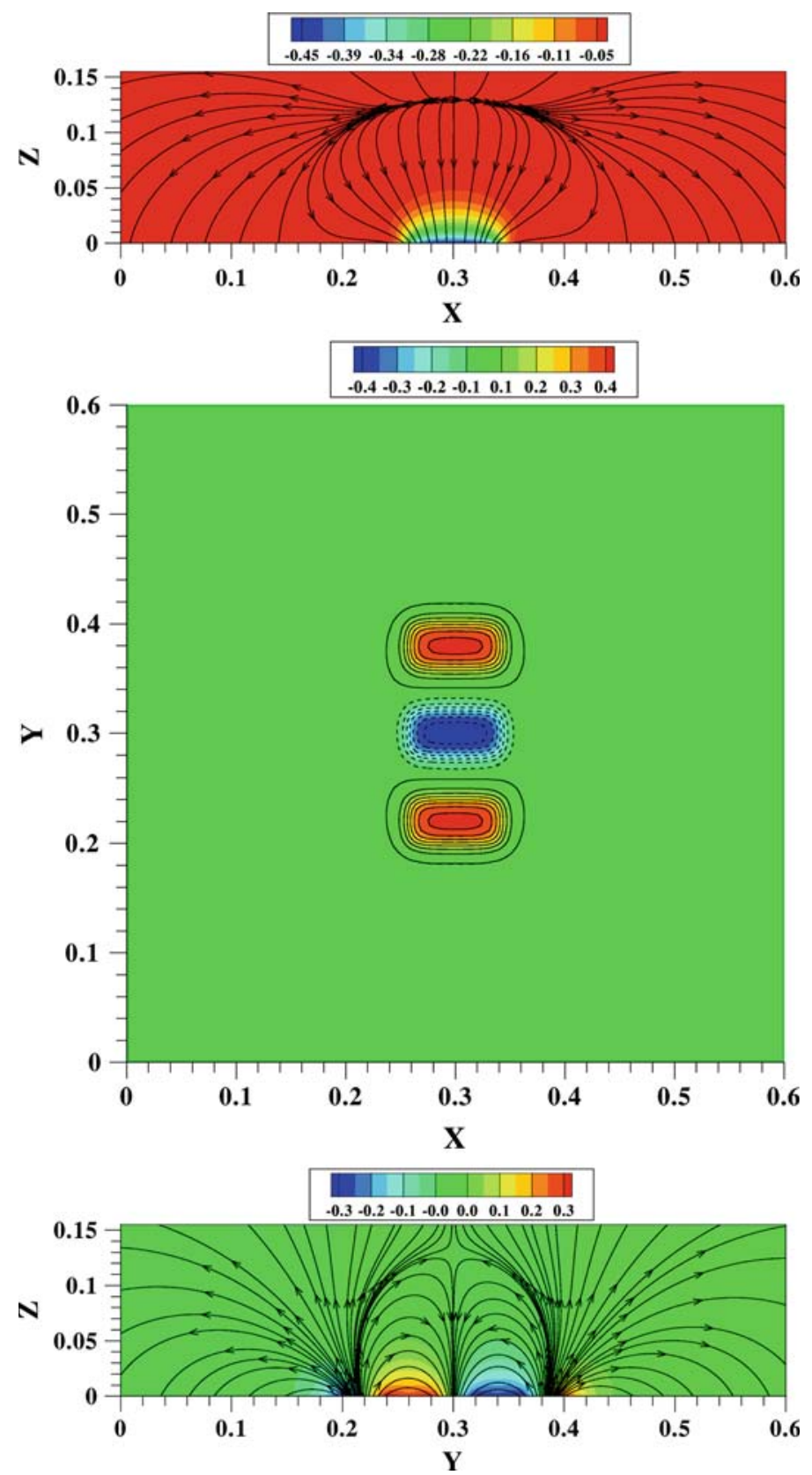

Fig. 2 The 3-magnets configuration: Distributions of the magnetic-flux lines and superimposed contours of the magnetic field components (in T): top $B_{z}$ in the $y / D=0.5$ plane, middle $B_{z}$ in the $z=0.001 \mathrm{~m}$ plane, bottom $B_{y}$ in the $x / L=0.5$ plane. The imposed electric field lines are distributed from left to right along the $x$-direction

regions with positive and negative values, are presented, Fig. 4(below/left). Two regions with positive vertical velocity components are generated along the lower and upper side walls. For the 3-magnet configuration, a central area is the most active region characterised by a positively oriented centreline distribution, and eight alternately directed (positive/negative) regions, Fig. 4(below/right).

The intensity of the electromagnetic forcing can be easily controlled by changing the intensity of the supplied DC current through the electrodes. This is demonstrated by showing the time-dependency of the maximum value of the velocity components for zero, weak $(I=1 \mathrm{~A})$ and strong $(I=10 \mathrm{~A}) \mathrm{DC}$ currents, Fig. 5. Besides producing higher velocities, stronger electric current also gives more intermittent signal, indicating the presence of a turbulent flow regime. It is interesting to note that the 2-magnet configuration, 

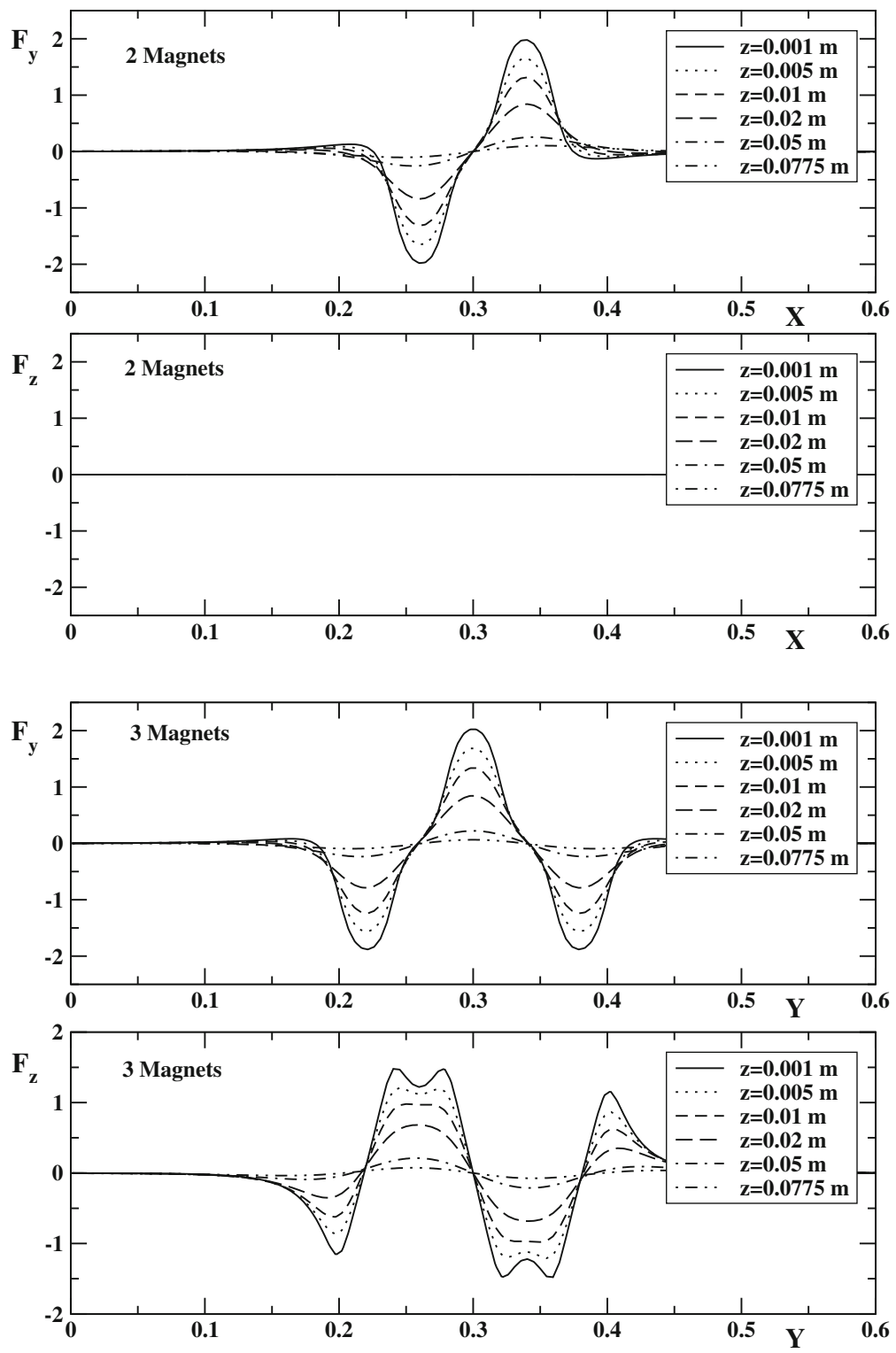

Fig. 3 The profiles of Lorentz force components (in $\left.\mathrm{N} / \mathrm{m}^{3}\right)$ along a horizontal line in the central vertical planes $(y / D=0.5$ and $x / L=0.5$ ) for different distances from the lower wall, for 2- and 3-magnets configurations, respectively. Applied DC current of $I=1 \mathrm{~A}$

Fig. 5 (left), generates significantly higher peak values in comparison to the 3-magnet configuration, Fig. 5 (right).

\subsection{Flow, turbulence and heat transfer}

In our earlier studies we performed a detailed validation of the LES approach by simulating the isothermal situation with identical configurations of both permanent magnets and electrodes, Kenjereš et al. [29,30]. Good agreement between experimentally obtained PIV velocity data and LES results at characteristic locations were obtained.

We studied the cases of pure turbulent thermal convection over a range of Rayleigh numbers, see Hanjalić and Kenjereš [12-14], Kenjereš and Hanjalić [19,21,22,25]. It was demonstrated that our simulations based 

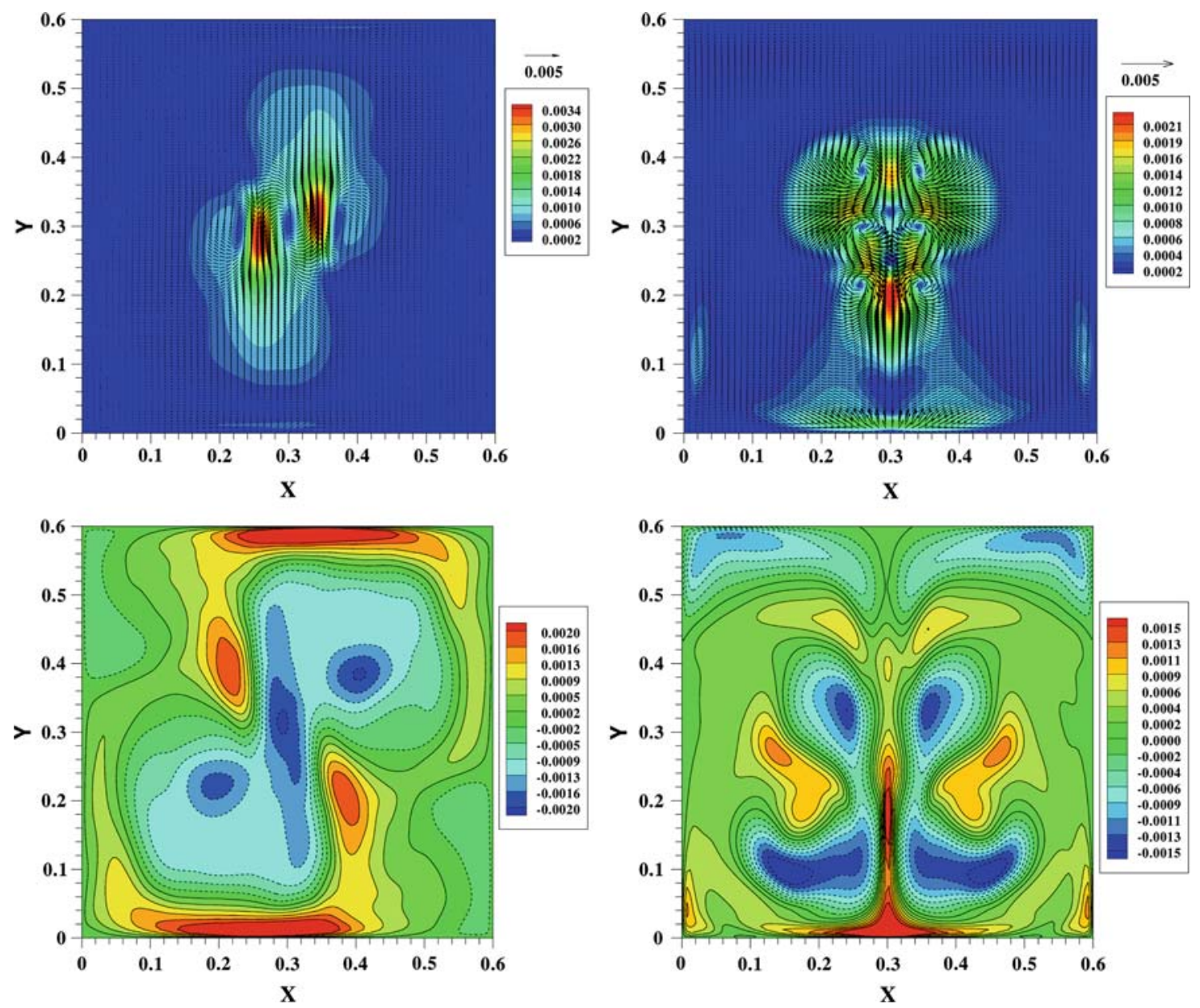

Fig. 4 Above- velocity vectors and velocity magnitude contours (in $\mathrm{m} / \mathrm{s}$ ) in a characteristic horizontal plane in the proximity of the lower wall $(z=0.001 \mathrm{~m})$; Below contours of the vertical velocity component (in $\mathrm{m} / \mathrm{s})$ in the central horizontal plane $(z / H=0.5)$; The pure electromagnetic forcing case for two configurations: 2-magnets (left), 3-magnets (right), both with applied DC current of $I=1 \mathrm{~A}$
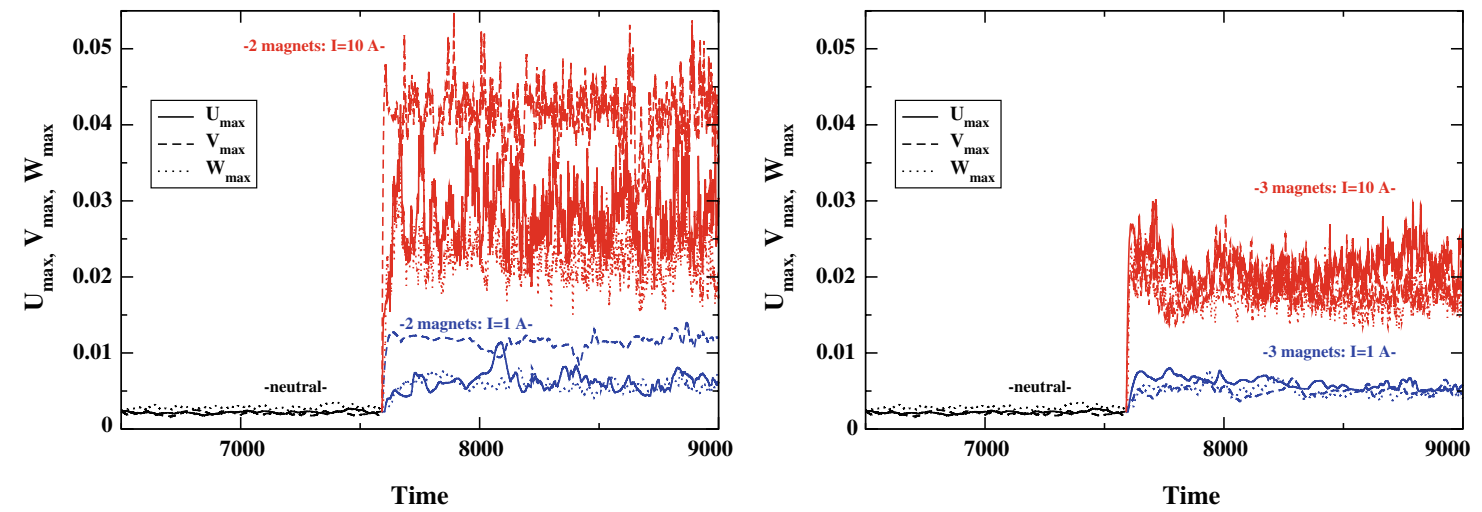

Fig. 5 The time-evolution of maximum velocity components for neutral, 2- and 3-magnets configurations and imposed electric DC currents of $I=1 \mathrm{~A}$ and $10 \mathrm{~A}$

on a T-RANS approach with a simplified second-moment closure for turbulent stress and turbulent heat fluxes produced a good agreement with available experimental, DNS and LES results in literature. In addition to the first and second-order long-time averaged statistics, our simulations also properly captured the dynamics 
of the large-convective structures. While T-RANS was only way to simulate ultra-turbulent flow regimes $\left(10^{12} \leq R a \leq 10^{17}\right)$, applications of LES proved to be accurate in predicting the wall-heat transfer for the intermediate values of $R a$ (up to $10^{9}$ ), Kenjereš and Hanjalić [25], Peng et al. [34]. In the present work, in order to create results for a neutral (without electromagnetic forcing) case, a pure turbulent thermal convection case is considered first. The integral wall-heat transfer is compared with available experimental studies of Chu and Goldstein [5] and Tanaka and Miyata [38], as well as with DNS studies of Kerr and Herring [32] -all addressing turbulent thermal convection of water in slender enclosures. The calculated value of $N u=15.35$ obtained from our long-time averaged LES data is in good agreement with DNS values of Kerr and Herring [32] of $N u=15.75$. Also, the agreement with available experiments under similar conditions is good. The experimentally obtained correlation from Tanaka and Miyata [38] gives $N u=0.14 R a^{0.29}=15.54$, and from Chu and Goldstein [5] gives $N u=0.183 R a^{0.278}=16.1$.

A complete overview of the performed simulations with electromagnetic forcing is shown in Table 1. Reynolds $(R e)$ and interactive $\left(N_{\mathrm{EMHD}}\right)$ numbers are given for specific magnetic configurations and imposed DC currents. The interactive number is expressed as $N_{\mathrm{EMHD}}=f_{\mathrm{EMHD}} H / \rho\left|\left\langle U_{i}\right\rangle\right|_{\max }^{2}$ where $f_{\mathrm{EMHD}}$ is the volumetric electromagnetic force, Thibault and Rossi [39]. It can be seen that different intensities of imposed DC currents can generate different flow regimes. Particularly challenging is the coexistence of different flow regimes in different parts of the tank. The strong electromagnetic forcing in the proximity of magnets can produce locally highly turbulent flow regimes, especially when the strongest DC current of $I=10 \mathrm{~A}$ is applied. Now we focus on the flow reorganisation and turbulence statistics for the combined effects of the thermal buoyancy and imposed electromagnetic forcing.

The superimposed stream-traces and temperature contours in characteristic horizontal planes located in the proximity of the lower wall and in the central plane, for different magnet configurations, are shown in Fig. 6. The stream-trace patterns for the neutral case in the proximity of the lower wall portray dominant horizontal movements without vortical components, see Fig. 6(above and left). In contrast to the neutral case, electromagnetic forcing for both 2- and 3-magnet configurations imposes significant vortical flow patterns inside the horizontal boundary layer. The imprints of the electromagnetic forcing can be easily associated with the underlying configurations of permanent magnets. The high local peaks of the local temperature are correlated with positive vertical velocity components (up-drafts), while the local minima are correlated with negative vertical velocity components (down-drafts). Distributions in the central horizontal plane $(z / H=0.5)$ also exhibit different behaviours. While the neutral case shows very few small vortical flow patterns, the 2- and 3-magnet configurations generate well-defined large vortical structures (Fig. 6(right)), even for applied DC strength of only $I=1 \mathrm{~A}$, large vortical structures. For the 2-magnet configuration, these vortical structures are located along the main diagonals of the enclosure with a strong central eddy. Hot- and cold-temperature regions are located in the centres of the large eddies. Also, along the side walls, small vertically oriented jets are created with typical hot regions, Fig.6(middle/right). The 3-magnet configuration creates a flow pattern with two dominant large eddies with a strong horizontal central jet in between, Fig. 6(below/right).

For identical configurations, distributions of temperature, stream-traces and horizontal velocity contours in the central vertical plane $(y / D=0.5)$ are shown in Fig. 7. For the neutral case, two large convective rolls are located in the central part of the enclosure, while two smaller secondary rolls are confined in the upper corners. Between these two central rolls, a descending thermal plume is located. This typical mushroom-shaped thermal plume closely resembles structures that are observed in Kenjereš and Hanjalić [21], Kerr [31] and Zhou et al. [48]. For the 2-magnet configuration, a strong swirling motion along the centreline reduces the horizontal extension of the downward thermal plume. As a consequence, the large central rolls are significantly reduced in size and are moved towards the lower side corners. The flow pattern for the 3-magnet configuration is characterised by a multiple-cell pattern generated by pump-in/pump-out forcing along the lower horizontal wall. The vertical central thermal plume is not visible anymore.

Contours of the horizontal velocity show symmetrical distributions along the centreline for the neutral and 2-magnet configurations, while the 3-magnet configuration exhibits asymmetrical distributions in the upper part of the enclosure, Fig. 7(right). For the neutral case, the contours of the horizontal velocity indicate four similar regions along the horizontal walls (both lower and upper wall) with identical magnitude and opposite signs. The 2-magnet forcing produces two large central regions and four distinct regions along the lower wall. In contrast to those distributions, the 3-magnet forcing creates dominant regions in the proximity of lower wall. The flow in the upper part of the enclosure is characterised by significantly smaller values of the horizontal velocity, confirming a boundary-layer type of electromagnetic forcing. 

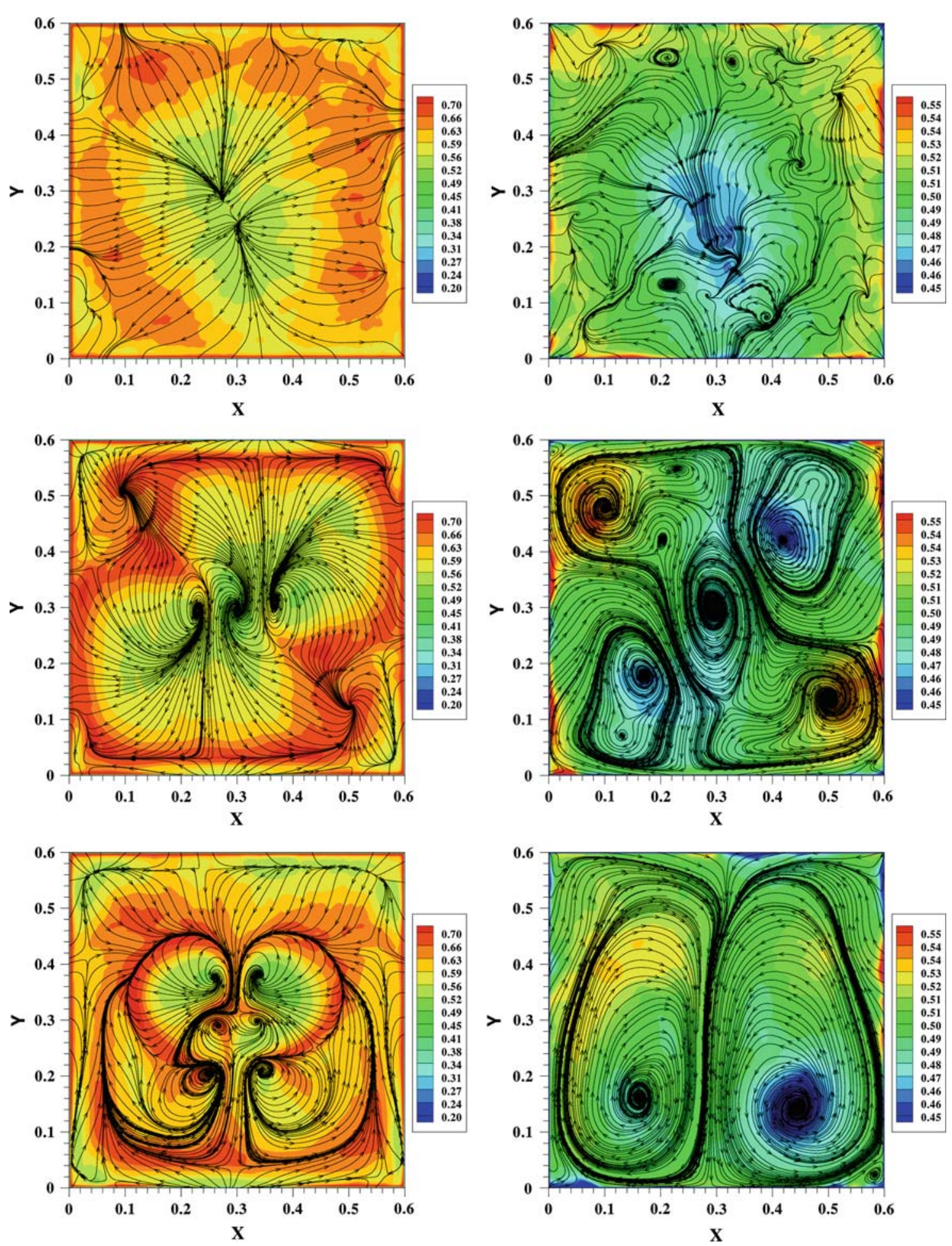

Fig. 6 The superimposed streamlines and temperature contours (for the long-time averaged fields) in characteristic horizontal planes, $z=0.005 \mathrm{~m}$ (left) and $z=0.0775 \mathrm{~m}$ (right). From top to bottom: neutral case, 2-magnets and 3-magnets configuration. Applied DC current of $I=1 \mathrm{~A}, \operatorname{Ra}=10^{7}, \mathrm{Pr}=7$

Contours of the resolved turbulent kinetic energy (TKE) in the central vertical plane $(y / D=0.5)$ are shown in Fig. 8(left). In the neutral case, the resolved TKE shows that the central part of the enclosure is associated with the largest velocity fluctuations. For the 2-magnet configuration, a distinct central region where a strongly 

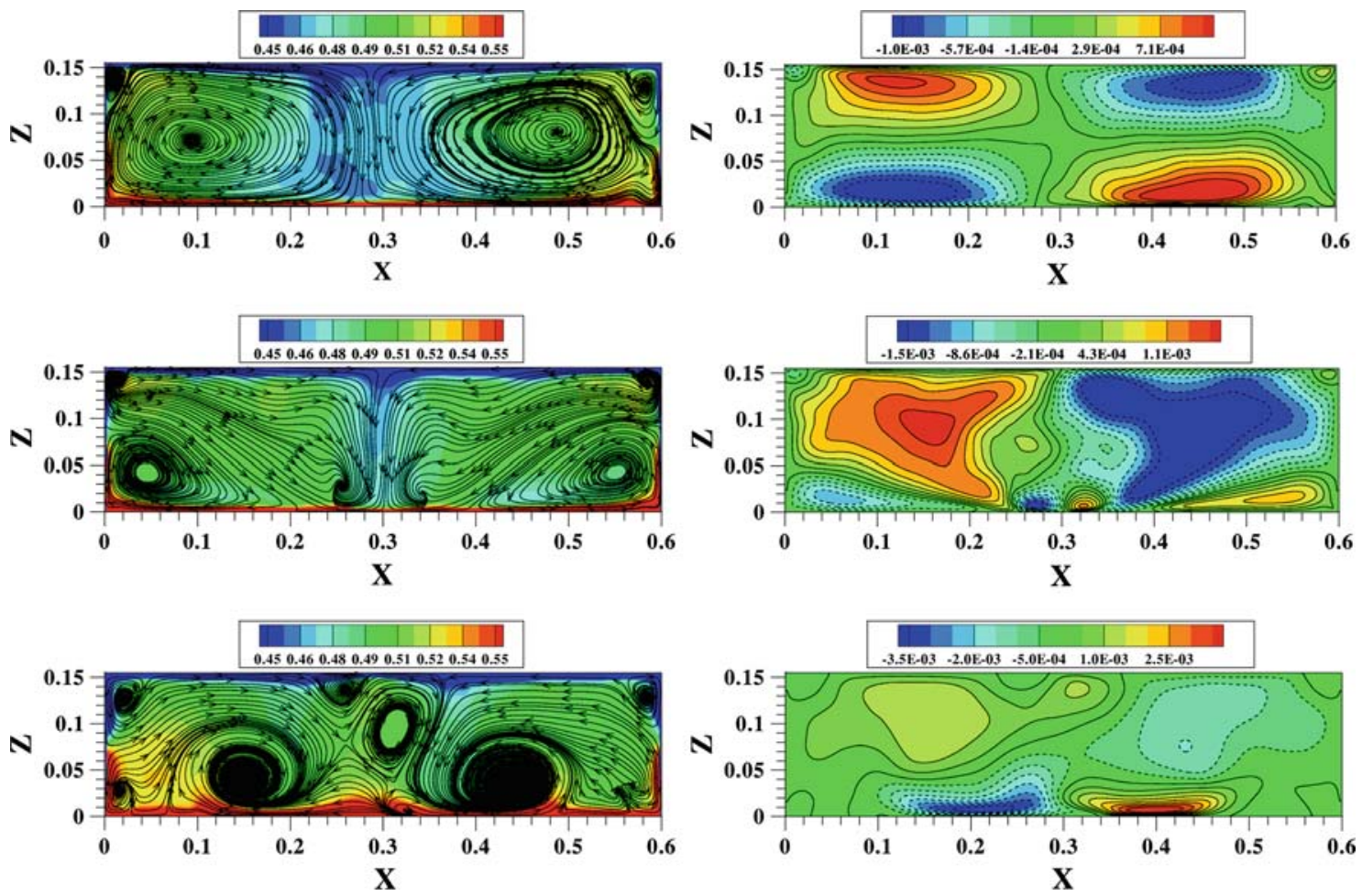

Fig. 7 Left- long-time averaged non-dimensional temperature $(\overline{\langle T\rangle} / \Delta T)$ field (contours) and superimposed stream-traces, Rightlong-time averaged horizontal velocity component (in $\mathrm{m} / \mathrm{s}$ ) all in the central vertical plane $(y / D=0.5)$ for different configurations: neutral case, 2-magnets configuration, 3-magnets configuration (from top to bottom), $I=1 \mathrm{~A}, \operatorname{Ra}=10^{7}, \mathrm{Pr}=7$. All $x$-, $y$ - and $z$-coordinates are given in (m). Note the difference of the scales (max and min values) for the horizontal velocity components, (right)

swirling flow is generated, shows the highest levels of TKE. Insulated patches of the significant turbulence activities are located in the proximity of the lower horizontal wall for the 3-magnet case.

Distributions of the temperature variance in the same central vertical plane are shown in Fig. 8(right). The horizontal boundary layers are places where the highest temperature fluctuations occur. The 2-magnet configuration clearly enhances the temperature fluctuations along the centreline. This is a consequence of the cold fluid stream that is carried by the strong swirling tornado-like motion (similar to that analysed in Yih [47]) that creates a vertically elongated thermal plume. It is noted that the 3 -magnet configuration significantly reduces the level of temperature fluctuations along the centreline. Some enhancements are observed along the horizontal boundary layers.

It is interesting to observe changes in flow and turbulence patterns for a stronger DC current of $I=10$ A, Fig. 9. A vortex merging of diagonally located eddies observed in Fig. 6 occurs for this stronger current. Compared to Fig. 6 for $I=1 \mathrm{~A}$, a significantly larger central eddy with strong turbulent core is created. In the upper right and the lower left corners, two smaller vortices are generated, as seen in Fig. 9(left). Also, for the 3-magnet configuration, forcing with stronger electric current creates more asymmetrically tilted eddies with respect to the central vertical line $(x=0.3 \mathrm{~m})$. The maximum values of the resolved turbulent kinetic energy is now located in the region between these two counter-rotating eddies, as shown in Fig. 9 (right).

The vertical profiles of resolved temperature, turbulent kinetic energy, temperature variance and horizontal and vertical turbulent stress are shown in Figs. 10, 11 and 12. It is important to note that these profiles are results of both long-time and spatial averaging. After reaching fully converged statistics of the first and second moments, additional plane averaging is performed, i.e. all points belonging to each horizontal plane are added and divided by the number of points in that plane. The vertical temperature profiles are characterised by very steep gradients in the proximity of the horizontal walls and a well-mixed central zone with almost uniform distribution, Fig. 10. In contrast to the situation where a uniform vertical magnetic field is imposed over the entire enclosure height and resulting vertical temperature profiles showed the appearance of a stable 

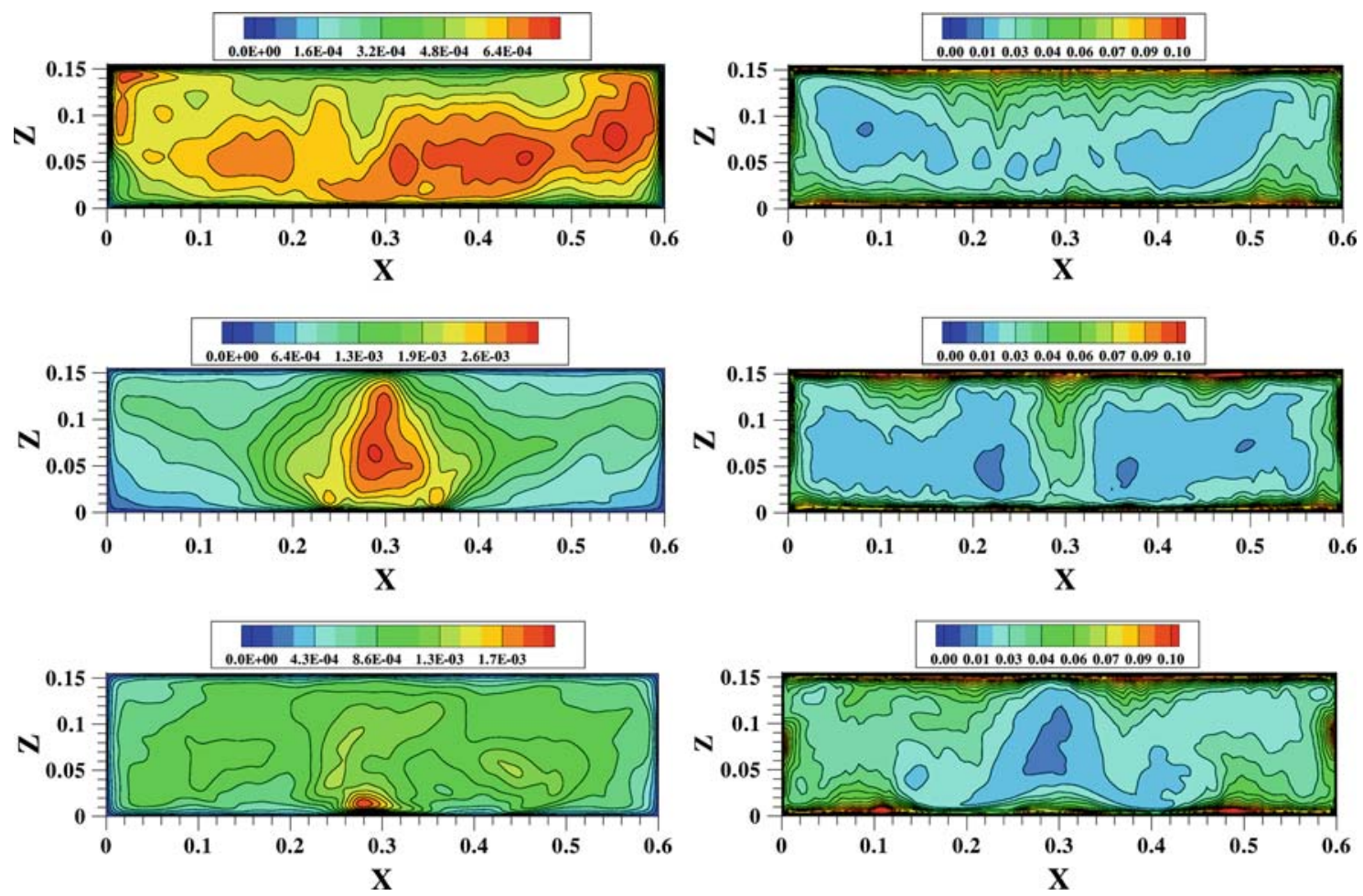

Fig. 8 Left- long-time averaged resolved turbulent kinetic energy $\left(k^{1 / 2}=\left[1 / 2 \overline{\left\langle u_{i}\right\rangle\left\langle u_{i}\right\rangle}\right]^{1 / 2}\right.$ in $\left.\mathrm{m} / \mathrm{s}\right)$, Right- long-time averaged temperature variance $\left(\overline{\langle\theta\rangle^{2}} / \Delta T\right)$ in the central vertical plane $(y=D / 2)$ for different configurations: neutral case, 2-magnets configuration, 3-magnets configuration (from top to bottom), $I=1 \mathrm{~A}, \operatorname{Ra}=10^{7}, \operatorname{Pr}=7$. Note the difference of the scales (max and min values) for the turbulent kinetic energy (left)
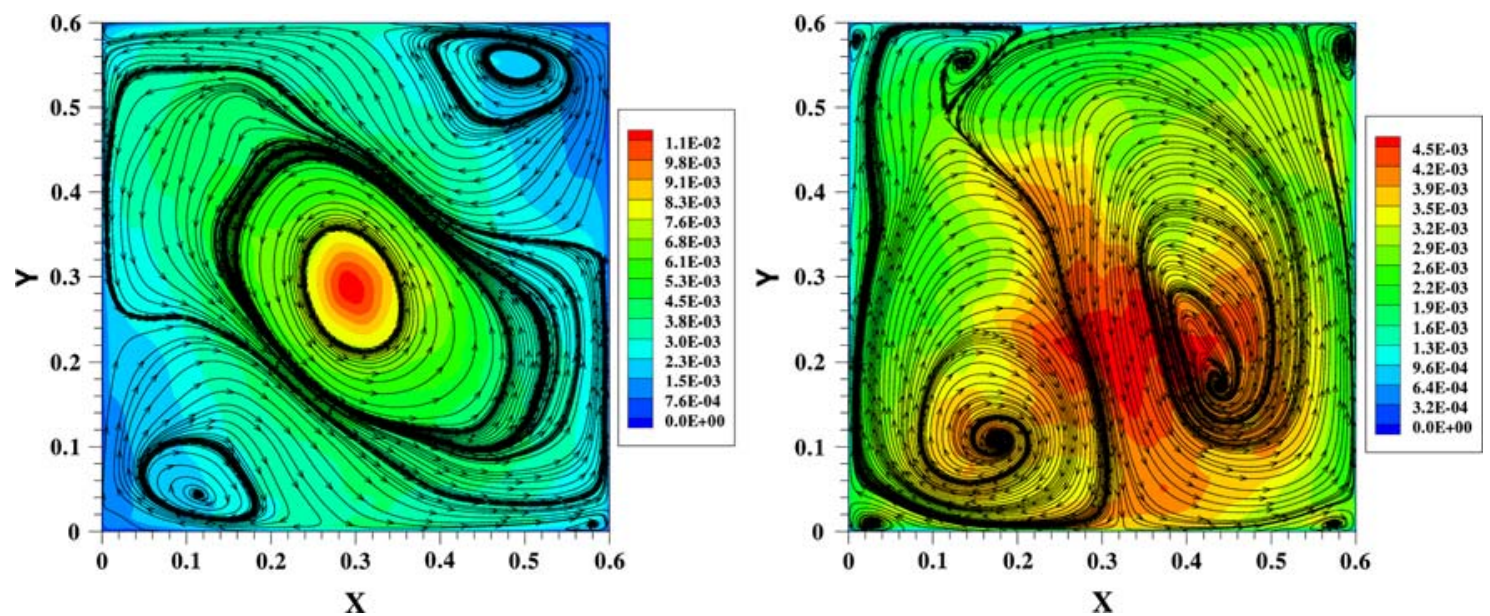

Fig. 9 The contours of the resolved turbulent kinetic energy and stream-traces in the central horizontal plane $z / H=0.5$ for strong electric current of $I=10$ A: left- 2-magnets configuration, right- 3-magnets configuration, $\operatorname{Ra}=10^{7}, \operatorname{Pr}=7$

stratified central region (as observed in Hanjalić and Kenjereš $[12,13]$ as well as in Cioni et al. [6]), for the local electromagnetic forcing an unstable stratification is present over the entire enclosure height. An interesting shift towards higher temperature values is observed for higher intensities of applied DC currents. Also, for the cases with electromagnetic forcing, a significant thinning of the thermal boundary layers along horizontal walls occurs (Fig. 10-zoom ins). 


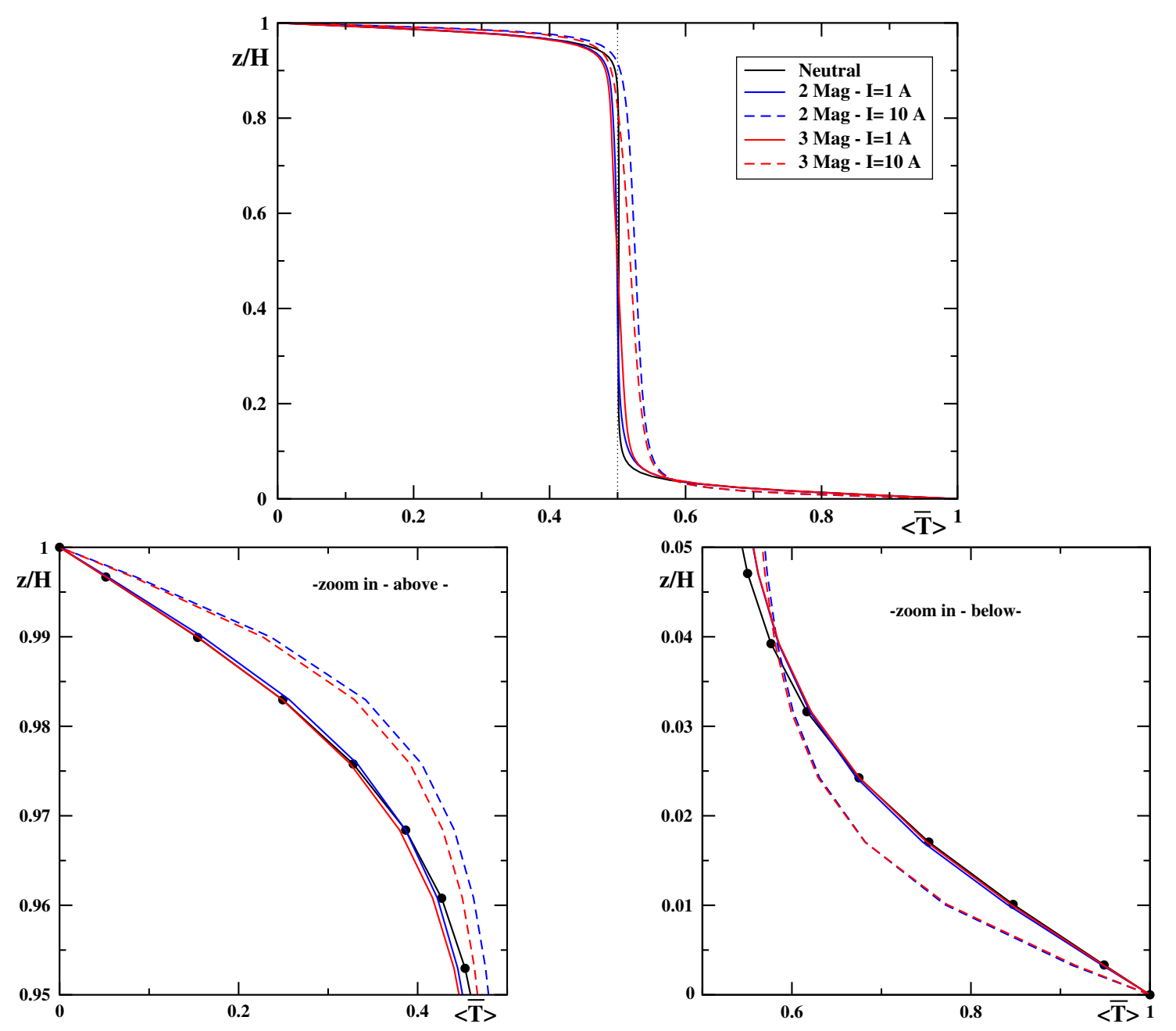

Fig. 10 The long-time and spatially averaged vertical temperature profile for different configurations and intensities of imposed electric current, $I=0,1,10$ A, respectively. The zoom-in of the near-wall regions in the proximity of the top (left) and bottom (right) walls. $R a=10^{7}, \operatorname{Pr}=7$

Electromagnetic forcing causes a dramatic increase in turbulent kinetic energy and in the asymmetry of its distribution with respect to the central horizontal line $(z / H=0.5)$, Fig. 11(above). In the configuration with 2-magnets and with highest applied DC current of $I=10$ A, almost an order of magnitude higher values of the peak TKE are generated in comparison with the neutral case. The asymmetry of the distributions is a consequence of the asymmetrical forcing, since only the lower part of the enclosure is subjected to the electromagnetic force.

When applying electromagnetic forcing, the peak values of the temperature variance (Fig. 11, below) are reduced in the proximity of the lower wall, whereas the peaks are enhanced in the region close to the upper wall. Also, significant reduction is observed in the central region for strong electric currents for both active configurations. The horizontal and vertical components of the resolved turbulent stress are shown in Fig. 12. It is obvious that application of electromagnetic forcing leads to a significant increase of the velocity fluctuations over the entire enclosure height. The horizontal component of the resolved turbulent stress is characterised by two peaks in the proximity of the horizontal walls, while the vertical component reach its maximum in the centre of the enclosure.

Finally, time evolutions of the integral Nusselt number over the lower and upper horizontal walls (for different configurations and imposed intensities of applied DC currents) are shown in Fig. 13. It is clearly demonstrated that the electromagnetic forcing enhances heat transfer for all considered situations. Additionally, for higher DC currents, strong intermittency (changes of the amplitude of time dependent variations) is 

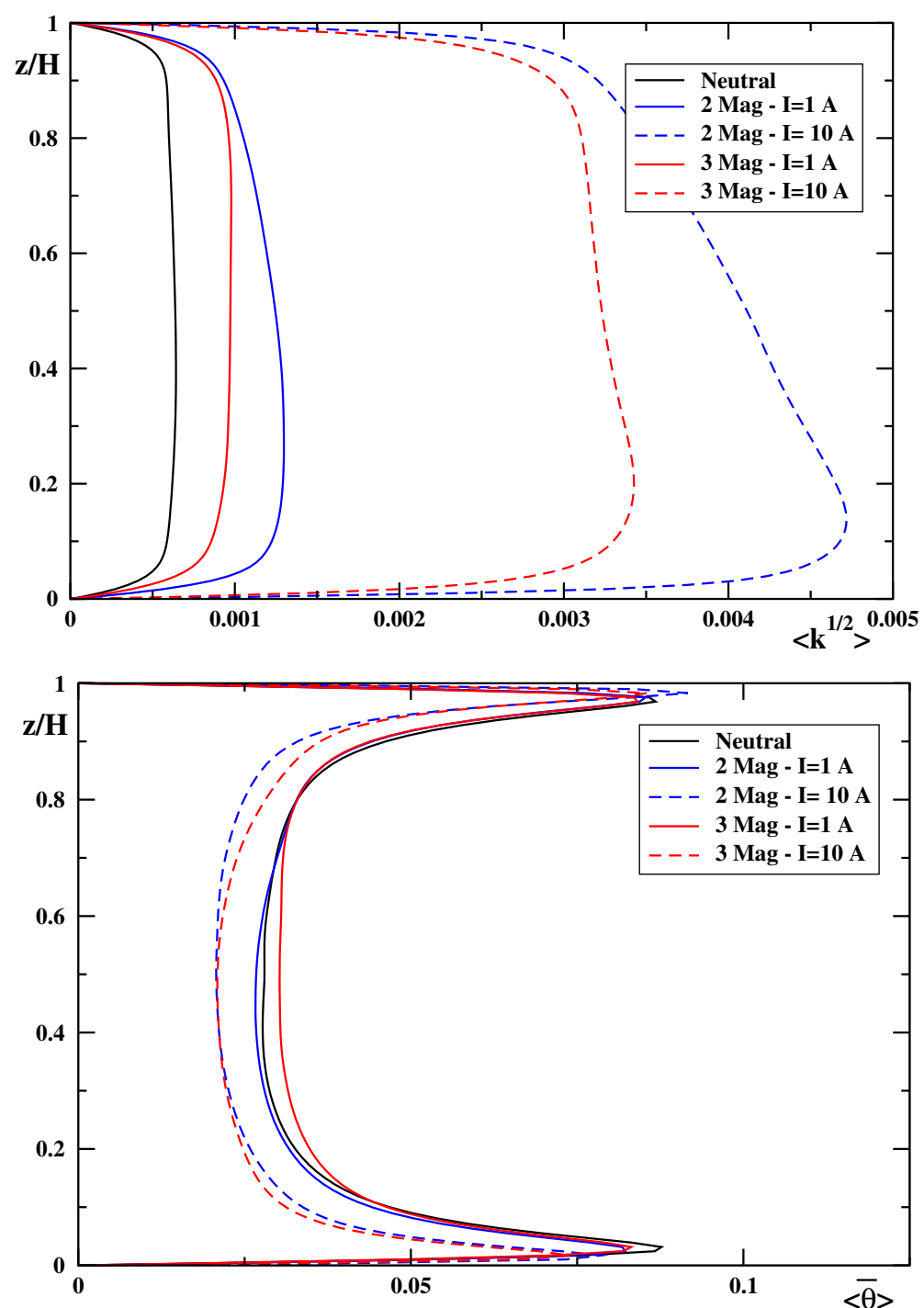

Fig. 11 The long-time and spatially averaged vertical profiles of resolved turbulent kinetic energy (above) and temperature variance (below) for different configurations and different intensities of imposed electric current. $\operatorname{Ra}=10^{7}, \mathrm{Pr}=7$, $I=0,1,10 \mathrm{~A}$

observed. In contrast to homogeneously applied magnetic fields which efficiently suppress flow, turbulence and heat transfer, targeted electromagnetic control, either locally confined in the horizontal boundary layers as in the 3-magnets configuration, or in the central part of the enclosure as in the 2-magnet configuration, intensifies the wall-heat transfer. The averaged values of Nusselt numbers are given in Table 1. It is interesting to note that the 2-magnet configuration is more efficient than the 3-magnet configuration for identical applied DC currents. The 2-magnet configuration produces heat transfer enhancements of 6 and $68 \%$ for weaker and stronger DC current, respectively, when compared to the neutral case. The 3-magnet configuration gives 2.3 and $63 \%$ Nusselt number increase for weak and strong DC current, respectively. The significance of the generated heat transfer enhancements for the stronger DC currents can be compared with other methods proposed in literature. In their experimental study of turbulent thermal convection inside of a vertical cylinder, Jin and Xia [18] applied periodic pulses of the input energy through the lower heated wall. The range of working parameters $5 \times 10^{7} \leq R a \leq 10^{8}$ and $\operatorname{Pr}=4.3$ was similar to parameters of the present study. This controlled variation of the lower wall temperature (referred as "kicked thermal turbulence") resulted in an increase up to 7\% in Nusselt number. Similarly, Uda et al. [42] reported experimental studies on heat transfer enhancements in annular turbulent flow $\left(4 \times 10^{3} \leq R e \leq 1.4 \times 10^{5}\right)$ of lithium subjected to 

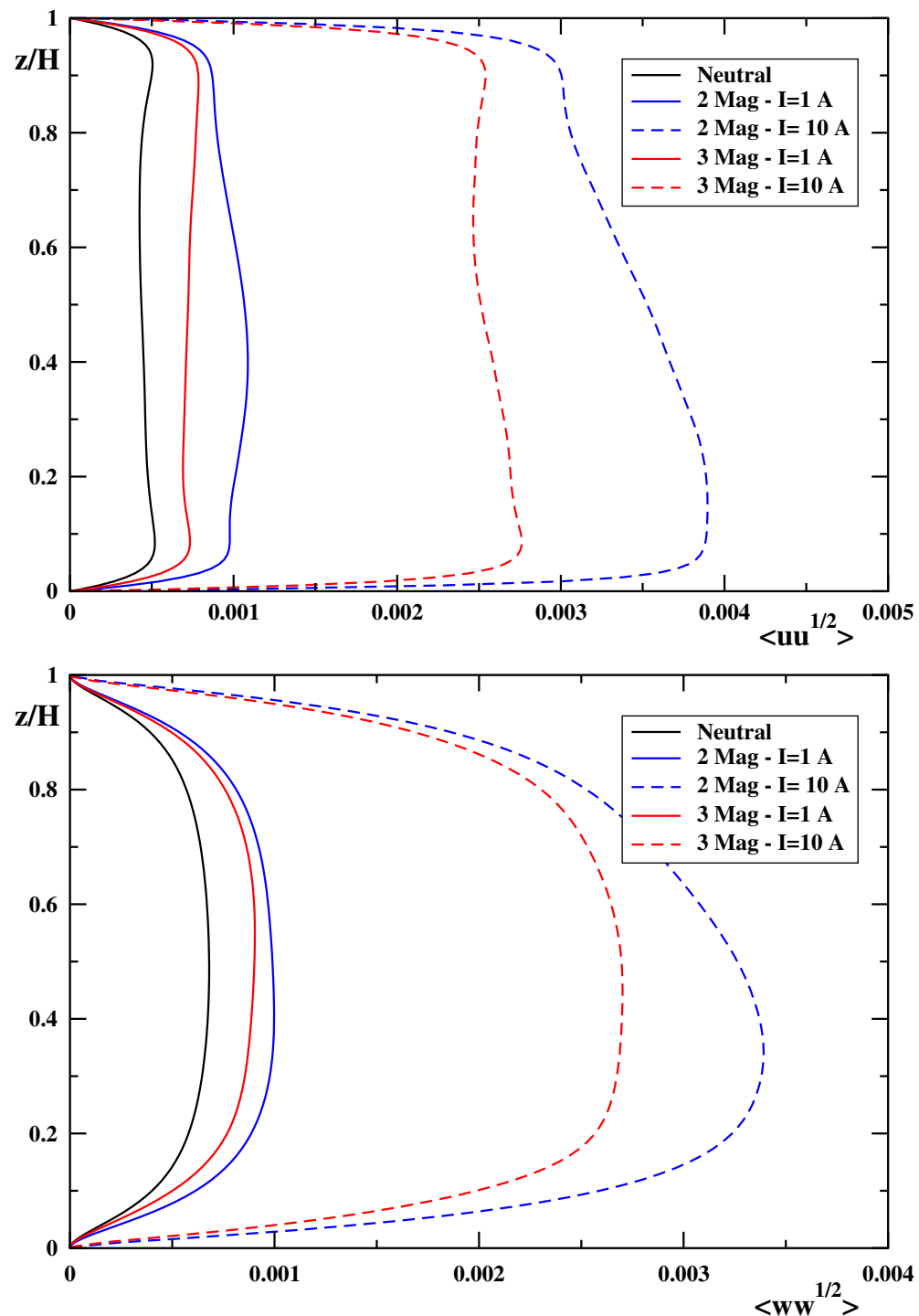

Fig. 12 The long-time and spatially averaged vertical profiles of the resolved velocity fluctuations (in m/s): horizontal (above), vertical (below) velocity component

transverse magnetic field $(0 \leq|B| \leq 0.8 \mathrm{~T})$. An increase of $5-10 \%$ of the Nusselt number was observed for $1 \leq H a^{2} / R e \leq 10$.

\section{Conclusions}

Numerical simulations of a turbulent thermal convection of an electrically conductive fluid in a slender enclosure heated from below and cooled from above, subjected to a locally distributed Lorentz force, revealed that electromagnetically promoted turbulence can significantly increase the wall-heat transfer. By simple reorganisation of small magnet arrays of opposite polarities, targeted control of flow and turbulence can be achieved. Electromagnetic forcing mainly imposed on the horizontal boundary layers along the lower wall is achieved by a 3-magnet configuration. A centrally located swirling flow is generated by applying a 2-magnet configuration. For both magnetic configurations, an enhancement of the wall-heat transfer is observed. While weak DC currents of $I=1$ A generate relatively modest heat transfer enhancements up to $6 \%$, a stronger DC current of $I=10$ A produces large increase of the wall-heat transfer up to $70 \%$. Beside its potential in 

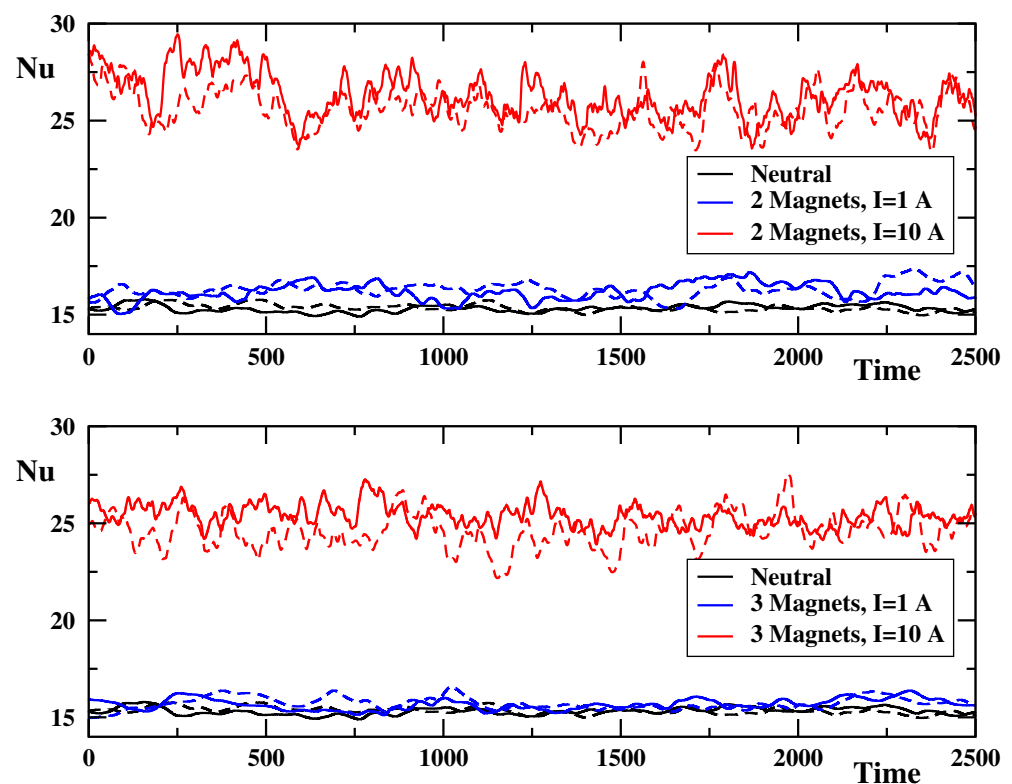

Fig. 13 Time evolutions of the integral Nusselt number over hot- (solid lines) and cold-horizontal walls (dashed lines) for different configurations and intensities of applied electric DC current, $I=0,1,10 \mathrm{~A}, \operatorname{Ra}=10^{7}, \operatorname{Pr}=7$

Table 1 Overview of simulated configurations with characteristic non-dimensional numbers

\begin{tabular}{|c|c|c|c|c|c|c|c|c|}
\hline \multirow[t]{2}{*}{ Applied DC current } & \multicolumn{2}{|l|}{ Re } & \multicolumn{2}{|l|}{$N_{\text {EMHD }}$} & \multicolumn{2}{|l|}{$\mathrm{Nu}$} & \multicolumn{2}{|l|}{$\Delta N u$} \\
\hline & $I=1 \mathrm{~A}$ & $I=10 \mathrm{~A}$ & $I=1 \mathrm{~A}$ & $I=10 \mathrm{~A}$ & $I=1 \mathrm{~A}$ & $I=10 \mathrm{~A}$ & $I=1 \mathrm{~A}$ & $I=10 \mathrm{~A}$ \\
\hline 2 Magnets & 1900 & 8250 & 1.3 & 0.7 & 16.27 & 25.75 & $+6.0 \%$ & $+68.0 \%$ \\
\hline 3 Magnets & 1100 & 4450 & 4.0 & 0.24 & 15.70 & 25.05 & $+2.3 \%$ & $+63.0 \%$ \\
\hline Neutral case & 500 & 500 & - & - & 15.35 & 15.35 & - & - \\
\hline
\end{tabular}

The magnetic field strength of permanent magnets is $\left|B_{0}\right|=1 \mathrm{~T}$ giving $H a=8\left(H a=\left|B_{0}\right| H \sqrt{\sigma / \rho \nu}\right)$. The Reynolds num-
ber is evaluated from the maximum values of instantaneous velocity field, $R e=\left\langle U_{i}\right\rangle_{\max } H / \nu$ and Nusselt number is $N u=$ $q /(v \Delta T / \operatorname{Pr} H)$

various industrial and technological applications, the concept of targeted electromagnetic control can be used for further fundamental studies of anisotropic turbulence.

Acknowledgments High-performance computing facilities were provided by the NWO/NCF and SARA Computational and Network services, Amsterdam, The Netherlands (www.sara.nl).

Open Access This article is distributed under the terms of the Creative Commons Attribution Noncommercial License which permits any noncommercial use, distribution, and reproduction in any medium, provided the original author(s) and source are credited.

\section{References}

1. Akoun, G., Yonnet, J.P.: 3D analytical calculation of the forces exerted between two cuboidal magnets. IEEE Trans. Magn. 20, 1962-1964 (1984)

2. Barleon, L., Burr, U., Mack, K.-J., Stieglitz, R.: Heat transfer in liquid metal cooled fusion blankets. Fusion Eng. Design 51(52), 723-733 (2000)

3. Burr, U., Müller, U.: Rayleigh-Bénard convection in liquid metal layers under the influence of a vertical magnetic field. J. Fluid Mech. 13(11), 3247-3257 (1984)

4. Cardoso, O., Marteau, D., Tabeling, P.: Quantitative experimental study of the free decay of quasi-two-dimensional turbulence. Phys. Rev. E 49(1), 454-465 (1994)

5. Chu, T.Y., Goldstein, R.J.: Turbulent convection in a horizontal layer of water. J. Fluid Mech. 60, 141-159 (1973)

6. Cioni, S., Chamumat, S., Sommeria, J.: Effect of a vertical magnetic field on turbulent Rayleigh-Bénard convection. Phys. Rev. E 62(4), R4520-R4523 (2000)

7. Dattarajan, S., Johari, H.: Control of a separating boundary layer with Lorentz force actuators. Phys. Fluids 20(045107), 1-15 (2008) 
8. Davidson, P.A.: Magnetohydrodynamics in materials processing. Annu. Rev. Fluid Mech. 31, 273-300 (1999)

9. Ferziger, J.H., Perić, M.: Computational Methods for Fluid Dynamics. Springer, Heidelberg (1999)

10. Gailitis, A.K., Lielausis, O.A.: On the possibility of drag reduction of a flat plate in an electrolyte. Appl. Magnetohydrodyn. Trudy Inst. Fis. AN Latv. SSSR, vol. 12, p. 143 (1961) (in Russian)

11. Grötzbach, G.: Spatial resolution requirement for direct numerical simulation of Rayleigh-Bénard convection. J. Comp. Phys. 49, 241-264 (1983)

12. Hanjalić, K., Kenjereš: Reorganisation of turbulence structure in magnetic Rayleigh-Bénard convection: a T-RANS study. J. Turbul. 1(8), 1-22 (2000)

13. Hanjalić, K., Kenjereš: T-RANS simulation of deterministic eddy structure in flows driven by thermal buoyancy and Lorentz force. Flow Turbul. Combust. 66(4), 427-451 (2001)

14. Hanjalić, K., Kenjereš: RANS-based VLES of thermal and magnetic convection at extreme conditions. J. Appl. Mech. Trans. ASME 73(3), 430-440 (2006)

15. Henoch, C., Stace, J.: Experimental investigation of a salt water boundary layer modified by an applied streamwise magnetohydrodynamic body force. Phys. Fluids 7(6), 1371-1383 (1995)

16. Honji, H., Ohkura, M., Ikehata, Y.: Flow patterns of an array of electromagnetically-driven cellular vortices. Exp. Fluids 23, 141-144 (1997)

17. Inaoka, K., Nakamura, K., Senda, M.: Heat transfer control of a backward-facing step flow in a duct by means of miniature electromagnetic actuators. Int. J. Heat Fluid Flow 25, 711-720 (2004)

18. Jin, X.L., Xia, K.Q.: An experimental study of kicked thermal turbulence. J. Fluid Mech. 606, 133-151 (2008)

19. Kenjereš, S., Hanjalić, K.: Transient analysis of Rayleigh-Bénard convection with a RANS model. Int. J. Heat Fluid Flow 20(3), 329-340 (1999)

20. Kenjereš, S., Hanjalić, K.: On the implementation of effects of Lorentz force in turbulence closure models. Int. J. Heat Fluid Flow 21(3), 329-337 (2000)

21. Kenjereš, S., Hanjalić, K.: Convective rolls and heat transfer in finite-length Rayleigh-Bénard convection: a two-dimensional study. Phys. Rev. E 62(6), 7987-7998 (2000)

22. Kenjereš, S., Hanjalić, K.: Numerical insights into flow structure in ultraturbulent thermal convection. Phys. Rev. E 66 (3), Art. No. 036307, 1-5 (2002)

23. Kenjereš, S., Hanjalić, K.: Numerical simulation of magnetic control of heat transfer in thermal convection. Int. J. Heat Fluid Flow 25(3), 559-568 (2004)

24. Kenjereš, S., Hanjalić, K., Bal, D.: A direct-numerical-simulation-based second-moment closure for turbulent magnetohydrodynamic flows. Phys. Fluids 16(5), 1229-1241 (2004)

25. Kenjereš, S., Hanjalić, K.: LES, T-RANS and hybrid simulations of thermal convection at high Ra numbers. Int. J. Heat Fluid Flow 27(5), 800-810 (2006)

26. Kenjereš, S., Hanjalić, K., Renaudier, S., Stefani, F., Gerbeth, G., Gailitis, A.: Coupled fluid-flow and magnetic-field simulation of the Riga dynamo experiment. Phys. Plasmas 13(12), Art. No. 122308, 1-14 (2006)

27. Kenjereš, S., Hanjalić, K.: Numerical simulation of a turbulent magnetic dynamo. Phys. Rev. Lett. 98 (10), Art. No. 104501, $1-4(2007)$

28. Kenjereš, S., Hanjalić, K.: Numerical insights into magnetic dynamo action in a turbulent regime. New J. Phys. 9, Art. No. 306, 1-29 (2007)

29. Kenjereš, S., Verdoold, J., Wibowo, A., Kleijn, C.R., Hanjalić, K.: Large eddy simulations of electromagnetically driven vortical flows. In: Palma, J.M.L.M., Lopes, A.S. (eds.) Springer Proceedings in Physics, vol. 117. Advances in Turbulence XI, pp. 656-658 (2007)

30. Kenjereš, S., Verdoold, J., Tummers, M.J., Hanjalić, K., Kleijn, C.R.: Numerical and experimental study of electromagnetically driven vortical flows. Int. J. Heat Fluid Flow 30(3), 494-504 (2009)

31. Kerr, R.M.: Rayleigh number scaling in numerical convection. J. Fluid Mech. 310, 139-179 (1996)

32. Kerr, R.M., Herring, J.R.: Prandtl number dependence of Nusselt number in direct numerical simulations. J. Fluid Mech. 419, 325-344 (2000)

33. Moreau, R.: Magnetohydrodynamics. Kluwer Academic Publishers, Dordrecht, The Netherlands (2000)

34. Peng, S.H., Hanjalić, K., Davidson, L.: Large-eddy simulation and deduced scaling analysis of rayleigh-Bénard convection up to $R a=10^{9}$. J. Turbul. 7(66), 1-29 (2006)

35. Rhie, C.M., Chow, W.L.: Numerical study of the turbulent flow past an airfoil with trailing-edge separation. AIAA J. 21(11), 1525-1532 (1983)

36. Shimomura, Y.: Large eddy simulation of magnetohydrodynamic turbulent channel flow under a uniform magnetic field. Phys. Fluids 3(12), 3098-3106 (1991)

37. Smagorinsky, J.: General circulation experiments with the primitive equations. I. The basic experiment. Mon. Weather Rev. 91, 99-164 (1963)

38. Tanaka, H., Miyata, H.: Turbulent natural-convection in a horizontal water layer heated from below. Int. J. Heat Mass Transf. 23, 1273-1281 (1980)

39. Thibault, J.P., Rossi, L.: Electromagnetic flow control: characteristic numbers and flow regimes of a wall-normal actuator. J. Phys. D Appl. Phys. 36(20), 2559-2568 (2003)

40. Rossi, L., Vassilicos, J.C., Hardalupas, Y.: Electromagnetically controlled multi-scale flows. J. Fluid Mech. 558, 207-242 (2006)

41. Rossi, L., Vassilicos, J.C., Hardalupas, Y.: Multiscale laminar flows with turbulentlike properties. Phys. Rev. Lett. 97 (14), 144501, 1-4 (2006)

42. Uda, N., Yamaoke, N., Horiike, H., Miyazaki, K.: Heat transfer enhancement in lithium annular flow under transverse magnetic field. Energy Convers. Manag. 43, 441-447 (2002)

43. van Driest, E.R.: On turbulent flow near a wall. J. Aero. Sci. 23, 1007-1011 (1956)

44. Verdoold, J., Rossi, L., Tummers, M.J., Hanjalić, K.: Towards electromagnetic control of thermal convection. In: Carlomagna, G.M., Grant, I. (eds.) Proceedings of the ISFCMV conference, Sorento, Italy, pp. 656-658. Optimage Ltd. (2003) 
45. Verdoold, J., Witte, P., Hoek, L., Tummers, M.J., Hanjalić, K.: Identification and electromagnetic control of structures in Rayleigh-Bénard convection. In: Humphrey, J.A.C., Gatski, T.B., Eaton, J.K., Friedrich, R., Kasagi, N., Leschziner, M.A. (eds.) Proceedings of the 4th Symposium on Turbulence and Shear Flow Phenomena, Virgina, USA, pp. 1-6 (2005)

46. Weier, T., Gerbeth, G., Mutschke, G., Lielausis, O., Lammers, G.: Control of flow separation using electromagnetic forces. Flow Turbul. Combust. 71, 5-17 (2003)

47. Yih, C.S.: Tornado-like flows. Phys. Fluids 19 (7), 076601, 1-6 (2007)

48. Zhou, Q., Sun, C., Xia, K.Q.: Morphological evolution of thermal plumes in turbulent Rayleigh-Bénard convection. Phys. Rev. Lett. 98, 074501, 1-4 (2007) 